the function of the spinal cord and prevent infection and other complications, an early surgical operation is fundamentally thought to be recommendable. Our method of operation for meningomyelocele and meningocele consisted of laminectomy of the proximal 1 or 2 vertebral arches to expose the normal dura in order to separate the dura from surrounding tissue distinctively and of suturing the ruptured dural margin exposed under electric stimulation.

In cases with an associated hydrocephalus first a shunt was conducted so. long as the surface of meningocele could be kept clean, and then operation for it was carried out.

Two cases of lipomeningocele presented no remarkable nervous symptoms. Although the present lesion is generally called lipoma, our cases of spina bifida with an associated dural rupture are often referred to as lipomeningocele.

The congenital dermal sinus was encountered in 2 cases. Since the dermal sinus proved to be a route of infection of myelomeningitis in our cases, it was removed at surgery. Therefore, much attention must be paid to the fact that the congenital dermal sinus very often gives rise to myelomeningitis as an invasion route of bacteria.

\title{
i-I0. Cranium Bifidum and Spina Bifida, their Treatment and remote Result
}

\author{
Katsuoki AKashi, Hiroo Chigasaki, Masakatsu Nagai and Keiichi Amano \\ Dept. of Neurosurgery, Univ. of Tokyo \\ Takeo KuwaHara \\ Dept. of Neurosurgery, Kanto Teishin Hospital
}

\section{i-11. Consideration of the Surgical Treatment for Myelomeningocele}

\author{
Tetsuro Miwa, Michitaka Kono, Masamichi Hasue, Morio Saito, \\ Yoshihisa Onodera, Toshitsugu Maki and Kenji TaKayasu \\ Department of Neurosurgery, Tokyo Medical College Hospital
}

Usually the surgical treatment of spina bifida cystica, especially the myelomeningocele and its significance are raising many controversies. Recently, in England, an early operation of the myelomeningocele (M.M.C.) is holding in period of 2 to 48 hours after birth and further active plastic operation of the M.M.C. is performing together with the atrio-ventricular shunt for combined hydrocephalus. Thus, the prevention of decrease of the intelligence and raise of survival rate were brought by this procedure. 